\title{
Initial Observation of Low-Energy Charged Particles by Satellite OHZORA (EXOS-C)
}

\author{
Toshifumi MukaI*, Nobuyuki Kaya**, Haruya Kubo*, Haruya \\ Matsumoto**, Tomizo ITOH*, and Kunio HiraO* \\ *The Institute of Space and Astronautical Science, Komaba, Meguro-ku, Tokyo, \\ Japan \\ **Faculty of Engineering, Kobe University, Rokko-dai, Nada-ku, Kobe, Japan
}

(Received March 4, 1985)

\begin{abstract}
The low-energy particle experiment (ESP) on the EXOS-C satellite was designed to measure the energy spectrum and the pitch-angle distribution of electrons over the energy range of $6 \mathrm{eV}$ to $16 \mathrm{keV}$ and positive ions from $200 \mathrm{eV}$ to $16 \mathrm{keV}$. The experiment has been successful and it has revealed global characteristics of ionospheric photoelectrons and magnetospheric energetic particles over a wide range of latitude, longitude, altitude $(350-850 \mathrm{~km})$ and local time. The precipitation of auroral particles is observed at latitudes higher than the trapping boundary of higher-energy particles. The electron precipitation pattern is diffuse in the dayside auroral region, whereas it is quite discrete on the nightside. The energy spectrum of ions precipitating into the auroral region is found to be generally harder than the corresponding electrons. Energetic electrons with energies of $10-100 \mathrm{keV}$ have also been detected at dusk hours near the equator. The nature of these electrons is not yet known. The energy spectrum of photoelectrons is found to have a peak in an energy range from 20 to $30 \mathrm{eV}$ and a sharp cutoff near $60 \mathrm{eV}$. However, the spectral shape in the energy range of 20 to $30 \mathrm{eV}$ is found to vary significantly depending on latitude, longitude and local time. A large peak at $20-30 \mathrm{eV}$ is observed occasionally over the region of the South-Atlantic geomagnetic anomaly.
\end{abstract}

\section{Introduction}

The EXOS-C (OHZORA) satellite was successfully launched on February 14, 1984 into a semi-polar orbit with inclination of $\sim 75^{\circ}$ and with the initial apogee and perigee, respectively, of $865 \mathrm{~km}$ and $353 \mathrm{~km}$. A low-energy particle experiment, named ESP, was installed on the satellite to study global characteristics of charged-particle distributions and interactions of particles with ambient plasma and waves.

The ESP instrument was designed to measure energy and pitch-angle distributions of electrons over the energy range from $6 \mathrm{eV}$ to $16 \mathrm{keV}$ and positive ions from $200 \mathrm{eV}$ to $16 \mathrm{keV}$, respectively. This instrument has been successfully operated and obtained the intensity and energy spectra of ionospheric photoelectrons in 
addition to the energetic particles over a wide range of latitude, longitude, altitude $(350-865 \mathrm{~km})$ and local time. It has been well recognized that particle observations in this energy range are essential for the understanding of the ionospheric and magnetospheric physics, and extensive measurements have been made (e.g., Meng, 1978; Voss and Smith, 1980; LeE et al., 1980a, 1980b). Most of the data obtained by the EXOS-C satellite are generally consistent with those obtained hitherto by others, but some peculiar phenomena have also been found out.

The purpose of this paper is to present characteristics of the instrument followed by a preliminary analysis of results which were obtained during the initial phase of satellite operation. The emphasis is laid on results of global features of ionospheric photoelectrons and energetic particles. A detailed discussion of auroral particle distributions is given in the other paper by KAYA et al. (1985, in this issue).

\section{Instrumentation}

The instrument ESP was designed to measure energy and pitch-angle distributions of electrons over the energy range from $6 \mathrm{eV}$ to $16 \mathrm{keV}$ and positive ions from $200 \mathrm{eV}$ to $16 \mathrm{keV}$, respectively. Figure 1 shows a schematic drawing of the instrument viewed from three orthogonal directions. It comprises three sensors (LE, HE and $\mathrm{HI}$ ) and a common electronics for control and processing of data. The LE sensor measures an energy distribution of low-energy electrons over the energy range from $6 \mathrm{eV}$ to $300 \mathrm{eV}$, while the $\mathrm{HE}$ and $\mathrm{HI}$ sensors measure
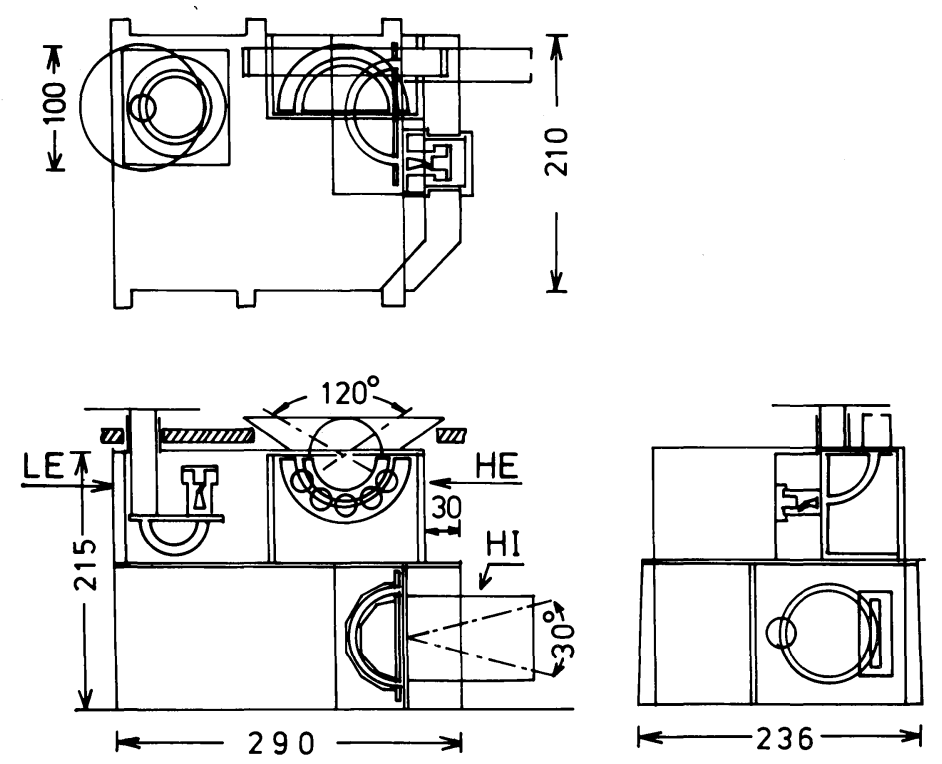

Fig. 1. Schematic drawing of the ESP instrument viewed from three orthogonal directions. 
higher-energy electrons and ions, respectively, from $200 \mathrm{eV}$ to $16 \mathrm{keV}$. Characteristics of each sensor are summarized in Table 1.

All three sensors have similar configurations in principle; each sensor consists of an inlet collimator, a spherical electrostatic analyzer and channeltron(s) (Galileo CEM 4039). Charged particles entering the analyzer through the collimator are deflected by the electric field $\left(E \propto 1 / r^{2}\right)$, and only those particles which have an appropriate energy can pass through the analyzer and impinge onto the channeltron. All channeltrons are shielded by aluminium of $\sim 1 \mathrm{gr} / \mathrm{cm}^{2}$ in order to reduce background count rate due to penetrating high-energy particles. The unique features of each sensor are described below.

(a) LE sensor

The main part of the LE sensor is surrounded by a high permiability metal in order to shield the external magnetic field. The collimator has a cylindrical shape with a circular electrode around the inlet part and is extruded from the satellite surface by $20 \mathrm{~mm}$. Its electric potential can be controlled by command to be $0,+1,+2,+5$, or +10 volts relative to the satellite in order to compensate the potential difference between ambient plasma and the satellite. The energy analyzer is hemispherical with inner and outer radii of $21.5 \mathrm{~mm}$ and $28.5 \mathrm{~mm}$, respectively, and has inlet and exit apertures of $3 \mathrm{~mm} \phi$. The potentials of inner and outer hemispheres are set to satisfy $R_{\mathrm{i}} V_{\mathrm{i}}+R_{\mathrm{o}} V_{\mathrm{o}}=0$ in order to minimize the fringing-field effect, where $R_{\mathrm{i}}$ and $R_{\mathrm{o}}$ are the radii of inner and outer hemispheres and $V_{\mathrm{i}}$ and $V_{\mathrm{o}}$ are the corresponding potentials relative to the collimator, respectively, ( $V_{\mathrm{i}}>0$ for electron measurement). A mesh with the potential of 5 volts below that of the outer hemisphere is inserted between the exit slit of the analyzer and the channeltron in order to suppress instrumental secondaries. Thus, although the energy is scanned from $4 \mathrm{eV}$ up to $300 \mathrm{eV}$ (see Table 2), the count data below $6 \mathrm{eV}$ provide background information (see Appendix). In order to measure more definitely the background count rate due to penetrating high-energy particles, the mesh potential is set at -80 volts for the step numbers 1 and 2 .

(b) HE sensor

Table 1. Characteristics of the ESP sensors.

\begin{tabular}{|c|c|c|c|}
\hline & \multicolumn{3}{|c|}{ Sensors } \\
\hline & LE & $\mathrm{HE}$ & $\mathrm{HI}$ \\
\hline $\begin{array}{l}\text { Analyzer } \\
\text { Energy range }\end{array}$ & $\begin{array}{c}\text { Hemispherical } \\
6-300 \mathrm{eV} \\
\text { electron }\end{array}$ & $\begin{array}{c}\text { Quadrispherical } \\
0.2-16 \mathrm{keV} \\
\text { electron }\end{array}$ & $\begin{array}{c}\text { Hemispherical } \\
0.2-16 \mathrm{keV} \\
\text { ion }\end{array}$ \\
\hline $\begin{array}{l}\text { Energy resolution } \\
\quad \Delta E / E\end{array}$ & $5 \%$ & $13 \%$ & $5 \%$ \\
\hline $\begin{array}{c}\text { Geometric factor }{ }^{(*)} \\
\mathrm{cm}^{2} \text { str } \mathrm{eV} / \mathrm{eV}\end{array}$ & $2.0 \times 10^{-5}$ & $1.3 \times 10^{-4}$ & $2.3 \times 10^{-4}$ \\
\hline Field of view & $6.2^{\circ}$ & $10^{\circ} \times 10^{\circ}$ & $10^{\circ} \times 30^{\circ}$ \\
\hline
\end{tabular}

(*) Per each channeltron. 
Table 2. Measurement energies and observation modes in the LE sensor.

\begin{tabular}{|c|c|c|c|c|c|c|c|c|c|}
\hline Step & Energy (eV) & A-mode & B-mode & C-mode & Step & Energy (eV) & A-mode & B-mode & C-mode \\
\hline 1 & $(3.56)^{*}$ & & & $\circ$ & 33 & 34.2 & & & \\
\hline 2 & $(3.82)^{*}$ & ○ & & $\circ$ & 34 & 36.7 & $\circ$ & & \\
\hline 3 & $(4.10)^{*}$ & & & $\circ$ & 35 & 39.4 & & & \\
\hline 4 & $(4.40)^{*}$ & $\circ$ & & $\circ$ & 36 & 42.3 & o & & \\
\hline 5 & $(4.72)^{*}$ & & & $\circ$ & 37 & 45.4 & & & \\
\hline 6 & $(5.07)^{*}$ & $\circ$ & & $\circ$ & 38 & 48.7 & 0 & & \\
\hline 7 & $(5.44)^{*}$ & & & $\circ$ & 39 & 52.3 & & & \\
\hline 8 & $(5.84)^{*}$ & ० & ○ & $\circ$ & 40 & 56.1 & $\circ$ & $\circ$ & \\
\hline 9 & 6.27 & & & $\circ$ & 41 & 60.2 & & & \\
\hline 10 & 6.73 & ० & & o & 42 & 64.6 & $\circ$ & & \\
\hline 11 & 7.22 & & & ○ & 43 & 69.3 & & & \\
\hline 12 & 7.75 & $\circ$ & & ० & 44 & 74.4 & $\circ$ & & \\
\hline 13 & 8.32 & & & ० & 45 & 79.9 & & & \\
\hline 14 & 8.92 & $\circ$ & & $\circ$ & 46 & 85.7 & $\circ$ & & \\
\hline 15 & 9.58 & & & o & 47 & 92.0 & & & \\
\hline 16 & 10.3 & ० & ० & ० & 48 & 98.7 & $\circ$ & $\circ$ & \\
\hline 17 & 11.0 & & & $\circ$ & 49 & 106. & & & \\
\hline 18 & 11.8 & 0 & & $\circ$ & 50 & 114. & o & & \\
\hline 19 & 12.7 & & & $\circ$ & 51 & 122. & & & \\
\hline 20 & 13.6 & $\circ$ & & $\circ$ & 52 & 131. & $\circ$ & & \\
\hline 21 & 14.6 & & & $\circ$ & 53 & 141. & & & \\
\hline 22 & 15.7 & $\circ$ & & o & 54 & 151. & $\circ$ & & \\
\hline 23 & 16.9 & & & o & 55 & 162. & & & \\
\hline 24 & 18.1 & $\circ$ & $\circ$ & $\circ$ & 56 & 174 & 0 & o & \\
\hline 25 & 19.4 & & & 0 & 57 & 187. & & & \\
\hline 26 & 20.8 & $0^{\prime}$ & & $\circ$ & 58 & 200 & 0 & & \\
\hline 27 & 22.4 & & & $\circ$ & 59 & 215 & & & \\
\hline 28 & 24.0 & $\circ$ & & $\circ$ & 60 & 231 & $\circ$ & & \\
\hline 29 & 25.8 & & & 0 & 61 & 248 & & & \\
\hline 30 & 27.7 & $\circ$ & & $\circ$ & 62 & 266. & o & & \\
\hline 31 & 29.7 & & & 0 & 63 & 285 & & & \\
\hline 32 & 31.9 & $\circ$ & $\circ$ & $\circ$ & 64 & 306. & ○ & ० & \\
\hline
\end{tabular}

*For background information.

The energy analyzer of the HE sensor is quadrispherical with inner and outer radii of $37.5 \mathrm{~mm}$ and $42.5 \mathrm{~mm}$, respectively, followed by five channeltrons. The analyzer is operated at usual balanced potentials; i.e., equal voltages of opposite polarity $\left(V_{\mathrm{i}}>0\right)$ are applied to the inner and outer spherical plates, respectively. The dispersion in the polar angle of the beam incidence results in the maximum spatial dispersion of the beam at the $90^{\circ}$ deflection in spherical electrostatic analyzers so that the exit position of the beam in a quadrispherical analyzer can be related directly to the polar angle of incidence. The channeltrons in the HE sensor are placed at the positions corresponding to the incident polar angles of $0^{\circ}, \pm 30^{\circ}$ and $\pm 60^{\circ}$, respectively. Thus, the HE sensor provides us with electron energy spectra at five different pitch-angles simultaneously. In addition, 
although the sensitivity of a usual quadrispherical analyzer has a dependence on the incident polar angle $\theta$ as $G \sim \cos \theta$ and hence decreases when $\theta \rightarrow \pm 90^{\circ}$, we have developed a sophisticated shaping collimator which gives identical view fields and geometric factors for five channeltrons without sacrificing sensitivities. Our quadrispherical analyzer has no slit at the entrance plane of the analyzer for defining the entrance area, and instead, the shaping collimator defines the entrance area of the analyzer as well as the field of view. The geometric factor for each channeltron is defined by the geometry of the collimator and the exit slit of the analyzer.

\section{(c) HI sensor}

The energy analyzer is a hemispherical one with the same inner and outer radii as those of the $\mathrm{HE}$ sensor, and it is operated also at the balanced potentials with $V_{\mathrm{i}}<0$. The field of view of the collimator is enlarged to $5^{\circ} \times 30^{\circ}$ in order to obtain a large geometric factor.

Figure 2 shows a schematic view of the EXOS-C satellite, where the LE and HE sensors are at the location designated ESP. The satellite has four solarcell paddles, and the bottom side of the spacecraft as illustrated in Fig. 2 is controlled to face the sun. Therefore, the LE and HE sensors view the anti-solar direction and are completely free from the EUV background due to the solar radiation. The HI sensor is also free from such background, since its view direction is perpendicular to the main axis of the satellite though it cannot be seen in Fig. 2.

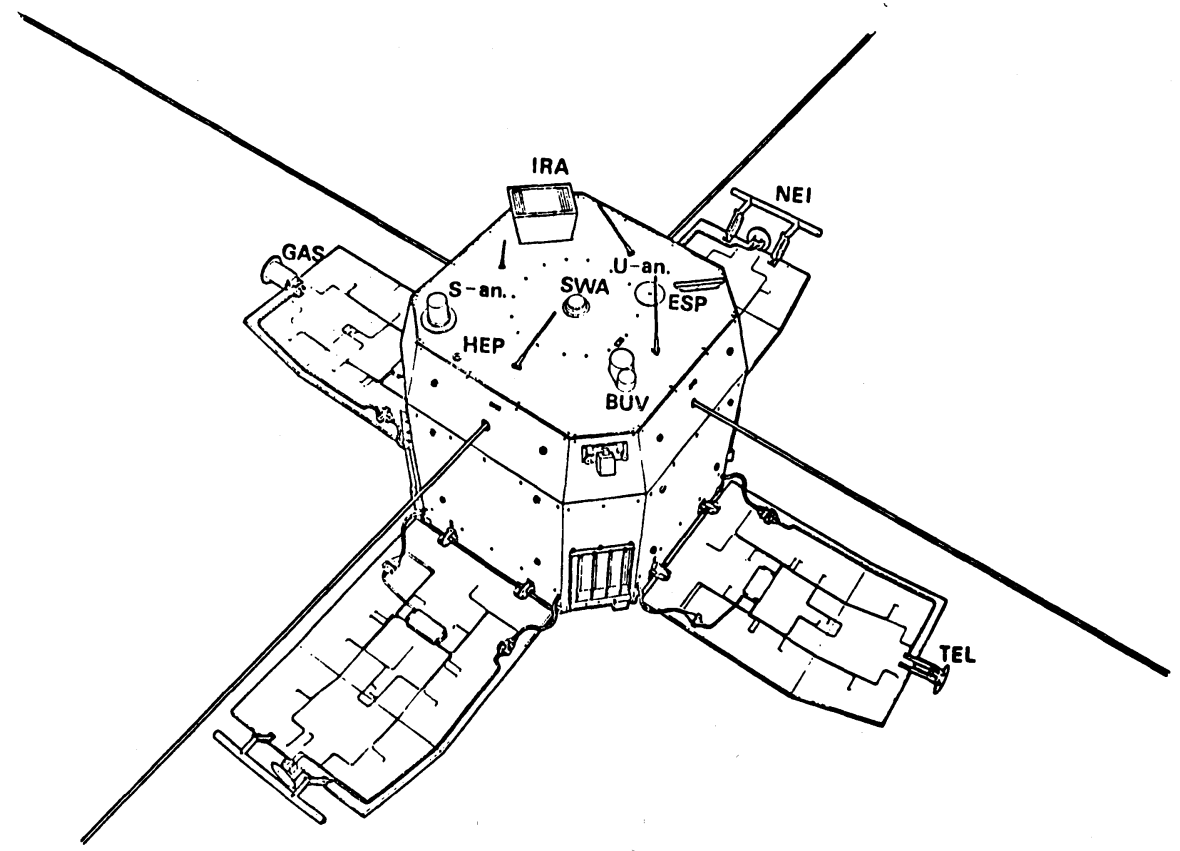

Fig. 2. Schematic view of the EXOS-C satellite. 
Figure 3 shows a block diagram of the instrument. As noted above, the instrument includes a total of seven channeltrons; one for LE, five for HE and one for HI. Each sensor has a high-voltage power supply for the channeltron(s) independently. The level of the high voltage can be selected by command in three steps of $3,3.5$ and $4 \mathrm{kV}$. Output charge pulses of each channeltron are amplified, discriminated, shaped and are counted by a 19-bit counter. Eight levels of pulse discrimination are prepared independently for each sensor in order to obtain pulse height distributions. The count data are compressed into 8-bit data and transmitted to the ground in the allocated PCM-telemetry words. The conversion of the compressed 8-bit word to the original counting can be made by the following relation.

$$
\begin{aligned}
& C=D \text { for } S=15, \text { or } \\
& C=2^{14-\mathrm{S}} \times(D+16) \text { for } S \neq 15,
\end{aligned}
$$

where $C$ is the original sample counts, and $D$ and $S$ are the decimal value of the upper and lower 4 bits of the 8-bit word, respectively.

Energy of measurement is scanned stepwise by applying stepped voltages (SV) to the analyzer plates. As shown in Fig. 3, the instrument includes two $\mathrm{SV}$ power supplies for energy scanning; one is common for both $\mathrm{HE}$ and HI, and another is for LE. Figure 4 shows energy-scanning modes for LE and HEI (HE and $\mathrm{HI}$ ), respectively. Tables 2 and 3 show the relationships among step numbers, measurement energies and scanning modes. It should be noted that the A-mode is a basic 32-step mode for both of LE and HEI, and that all results presented in subsequent sections have been obtained in the A-mode. The B-mode is a high time resolution mode which sacrifices the energy resolution, and the $\mathrm{C}$ and $\mathrm{D}$ modes for HEI are prepared for detecting modulated fluxes.

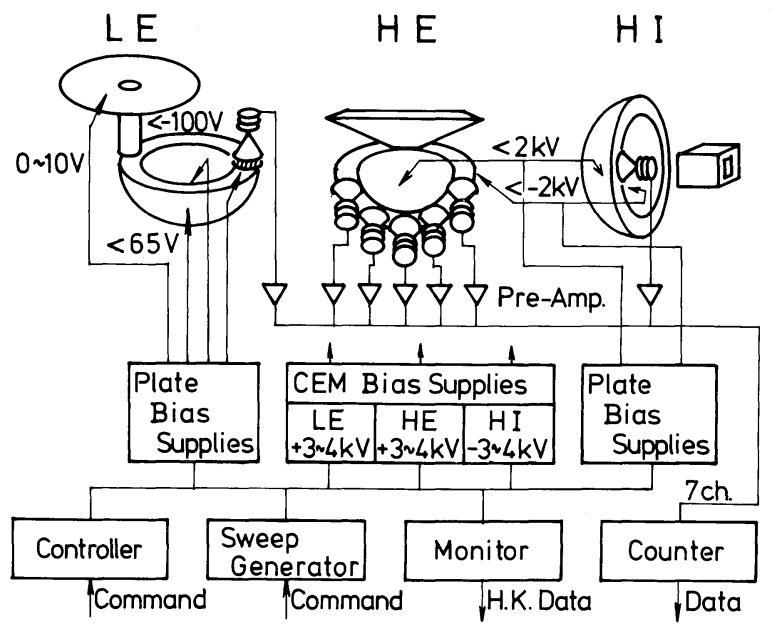

Fig. 3. Block diagram of the ESP instrument. 

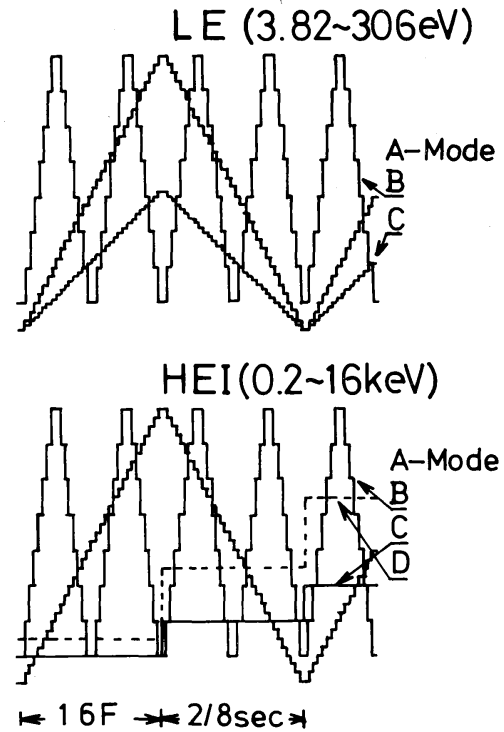

Fig. 4. Energy scanning modes.

The differential particle flux $f(E)$, particles $/ \mathrm{cm}^{2}$ sec str eV, can be obtained from the observed counts $C$ by $f(E)=C /\langle G \cdot \Delta E\rangle \cdot \tau^{\cdot} \varepsilon$, where $\langle G \cdot \Delta E\rangle$ is an energygeometric factor of the analyzer in $\left[\mathrm{cm}^{2} \mathrm{str} \mathrm{eV}\right], \tau$ is the sampling time and $\varepsilon$ is the detection efficiency. For an analyzer of the type used in this instrument, the energy resolution, $\Delta E / E$ is constant over the energy range of measurement, where $E$ is the center energy of transmitted particles and $\Delta E$ is the energy bandwidth, respectively. To estimate $\langle G \cdot \Delta E\rangle$ it is necessary to know the transmission function of the analyzer which is a complex function of the inlet position, twodimensional incidence angle and energy of incident particles. Characteristics of each sensor were derived by numerical fitting of the transmission function obtained experimentally in the pre-flight calibration test and are summarized in Table 1. Figure 5 shows an example of transmission curves for the HE sensor. It can be seen that the fitted curves agree well with experimental results.

\section{Instrument Performance in Orbit}

Operation of the ESP experiment commenced about 4 weeks after the launch, at which time all high-voltage power supplies were turned on successfully, and it was confirmed that the whole instrument of ESP operated normally. In this section we describe the performance of the instrument in orbit for better understanding of the observational results.

\subsection{Channeltron performance}

A pulse height distribution of each channeltron output was examined by 
Table 3. Measurement energies and observation modes in the $\mathrm{HE}$ and $\mathrm{HI}$ sensors.

\begin{tabular}{|c|c|c|c|c|c|}
\hline Step & Energy $(\mathrm{keV})$ & A-mode & B-mode & C-mode & D-mode \\
\hline 1 & $(0.20)^{*}$ & $\circ$ & & & \\
\hline 2 & $(0.23)^{*}$ & $\circ$ & & & \\
\hline 3 & $(0.27)^{*}$ & $\circ$ & & & \\
\hline 4 & $(0.31)^{*}$ & $\circ$ & $\circ$ & $\circ$ & \\
\hline 5 & 0.35 & $\circ$ & & & \\
\hline 6 & 0.41 & $\circ$ & & & ○ \\
\hline 7 & 0.47 & ० & & & \\
\hline 8 & 0.54 & $\circ$ & $\circ$ & o & \\
\hline 9 & 0.62 & $\circ$ & & & \\
\hline 10 & 0.71 & $\circ$ & & & \\
\hline 11 & 0.82 & $\circ$ & & & \\
\hline 12 & 0.95 & $\circ$ & $\circ$ & $\circ$ & \\
\hline 13 & 1.09 & $\circ$ & & & \\
\hline 14 & 1.26 & ० & & & $\circ$ \\
\hline 15 & 1.45 & $\circ$ & & & \\
\hline 16 & 1.67 & $\circ$ & ० & $\circ$ & \\
\hline 17 & 1.92 & $\circ$ & & & \\
\hline 18 & 2.21 & $\circ$ & & & \\
\hline 19 & 2.55 & ○ & & & \\
\hline 20 & 2.93 & $\circ$ & o & $\circ$ & \\
\hline 21 & 3.38 & ○ & & & \\
\hline 22 & 3.89 & $\circ$ & & & ० \\
\hline 23 & 4.48 & $\circ$ & & & \\
\hline 24 & 5.16 & $\circ$ & 0 & $\circ$ & \\
\hline 25 & 5.95 & $\circ$ & & & \\
\hline 26 & 6.85 & $\circ$ & & & \\
\hline 27 & 7.89 & $\circ$ & & & \\
\hline 28 & 9.09 & $\circ$ & o & $\circ$ & \\
\hline 29 & 10.5 & $\circ$ & & & \\
\hline 30 & 12.1 & $\circ$ & & & ○ \\
\hline 31 & 13.9 & $\circ$ & & & \\
\hline 32 & 16.0 & $\circ$ & 0 & $\circ$ & \\
\hline
\end{tabular}

*: Overlapped with the LE measurement.

inter-comparison among the data obtained at different levels of pulse discrimination. The result indicated that all channeltrons had sufficient gains so that they were operating in the well-saturated range at the lowest level of the high voltage. It was also decided that the discrimination level of the fifth level was suitable for all channeltrons. No significant degradation in the channeltron gain has been detected in 5 months after the launch.

\subsection{LE bias}

As noted in section 2, the potential (LE bias) of the inlet collimator of the LE sensor can be selected by command to be one of $0,+1,+2,+5$ and +10 volts relative to the satellite. It has been determined by examination of the ob- 


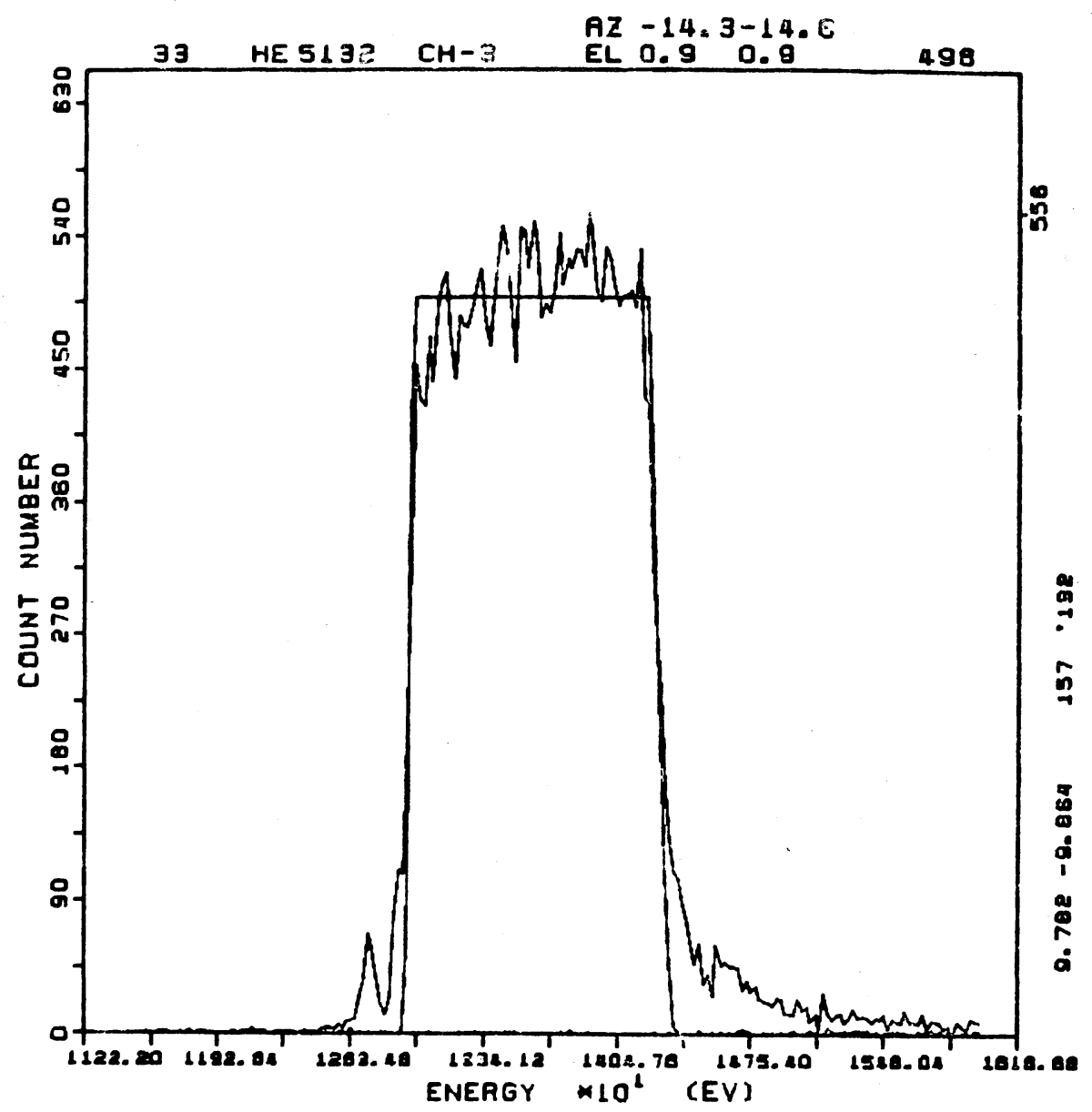

(a)

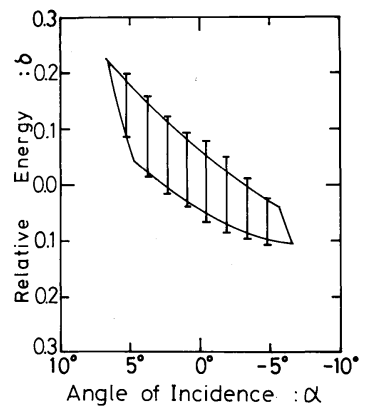

(b)

Fig. 5. Examples of transmission curves for HE-3 (a) Energy response. The zig-zag line shows an experimental result, and the smooth rectangular response is numerical fitting. (b) Energyangle response. The bars show responses obtained experimentally, and the contour curve shows a numerical result, respectively. 
tained data that the LE bias of 1-volt level is the most suitable. The measured energies of the LE data are relative to this LE bias.

\subsection{Background count rate and its subtraction}

As noted in section 2, the LE sensor has background channels (steps) in the energy scanning. Therefore, the background count rate has been subtracted easily from the signal count rate, although the background has been negligibly small except in the South-Atlantic anomaly region. Other two sensors have no specified channels for background information, but the data have indicated that the background count rate is negligibly small also in the HI sensor except in the South-Atlantic anomaly region. Therefore, no background correction has been made in the HI data. The dark counts in the HE data, however, have been found to be greater than expected, and the count rate corresponding to $2 \times 10^{6}$ $\mathrm{els} / \mathrm{cm}^{2} \mathrm{sec}$ str $\mathrm{keV}$ at $1 \mathrm{keV}$ has been subtracted from $\mathrm{HE}$ data in normal circumstances. We believe that this does not produce a problem for investigation on the auroral electron precipitation. Validity of the background subtraction from the HE data has been examined by comparing the result with the LE data in the overlapping energy range of 200-300 eV. Figure 6 shows an example of data comparison between the LE and HE data which were obtained during stable precipitation in the dayside auroral zone at Rev. 00974. It can be seen that both data show a good agreement.

\section{Observational Results and Discussion}

The ESP experiment has yielded global characteristics of low energy chargedparticle distributions over a wide range of latitude, longitude, altitude (350-865 $\mathrm{km}$ ) and local time. In this section some typical examples of data are presented and discussed. Here the term "typical" means that similar features are observed frequently in many orbits other than the ones presented here.

Figures 7, 8 and 9 show global patterns of charged-particle distributions obtained from the ESP and HEP (high energy particle) experiments at Revs. 00835, 00944 and 00953, respectively, in moderate geomagnetic activity; $K p$ indices were 2-, 3- and 2 at Revs. 00835, 00944 and 00953, respectively. In each figure, the upper five panels show the energy-time spectrograms of the LE, HE-3, HE-5, HE-1 and HI data from ESP, while the lower two pannels show the integrated flux obtained from the S-1 sensor of HEP. The corresponding pitch angles are also shown. Note that the $0^{\circ}$-pitch angle means precipitation in the northern hemisphere, whereas the $180^{\circ}$-pitch angle does so in the southern hemisphere. In the energy-time spectrogram the ordinate is particle energy and the abscissa is UT. Gray-scaled intensity indicates the count rate which is proportional to the differential energy flux. The satellite location is given in the bottom rows.

The HE and HI data show several characteristic zones of energetic particle distributions; auroral zones in the southern and northern hemispheres, the region 


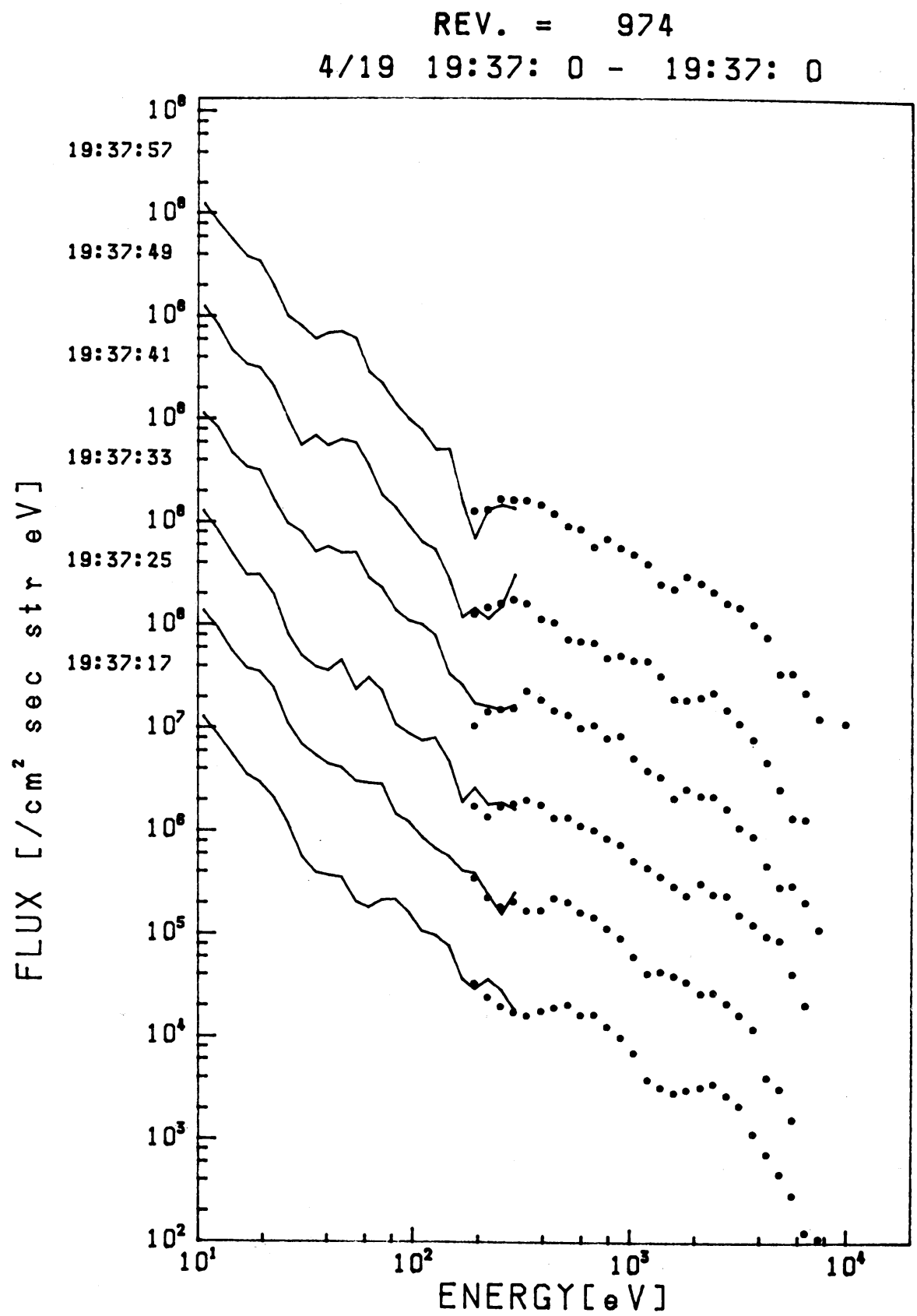

Fig. 6. Intercomparison between the $\mathrm{LE}$ and $\mathrm{HE}$ results. 


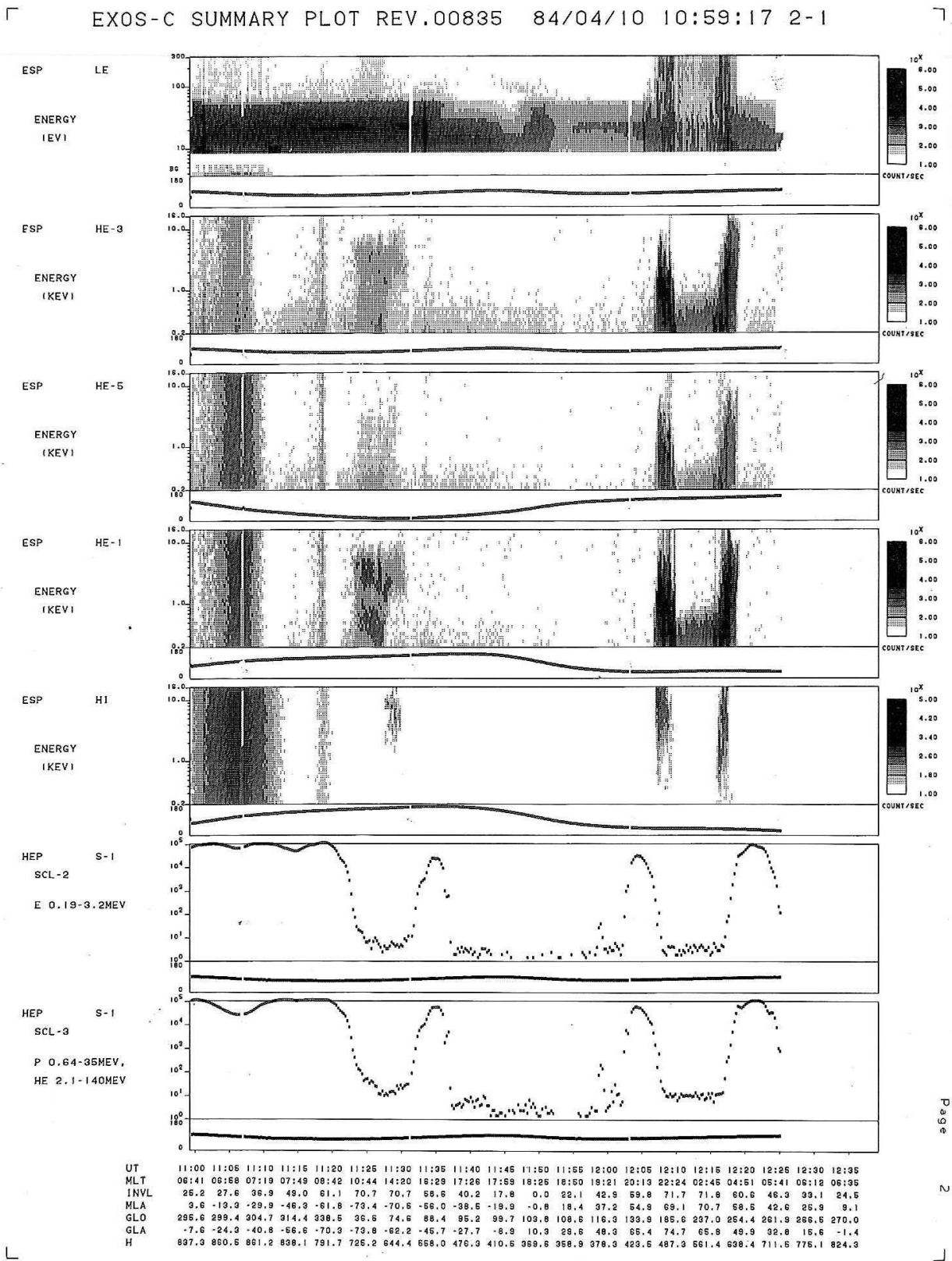

Fig. 7. Global patterns of charged particle distributions obtained at Rev. 00835 . 


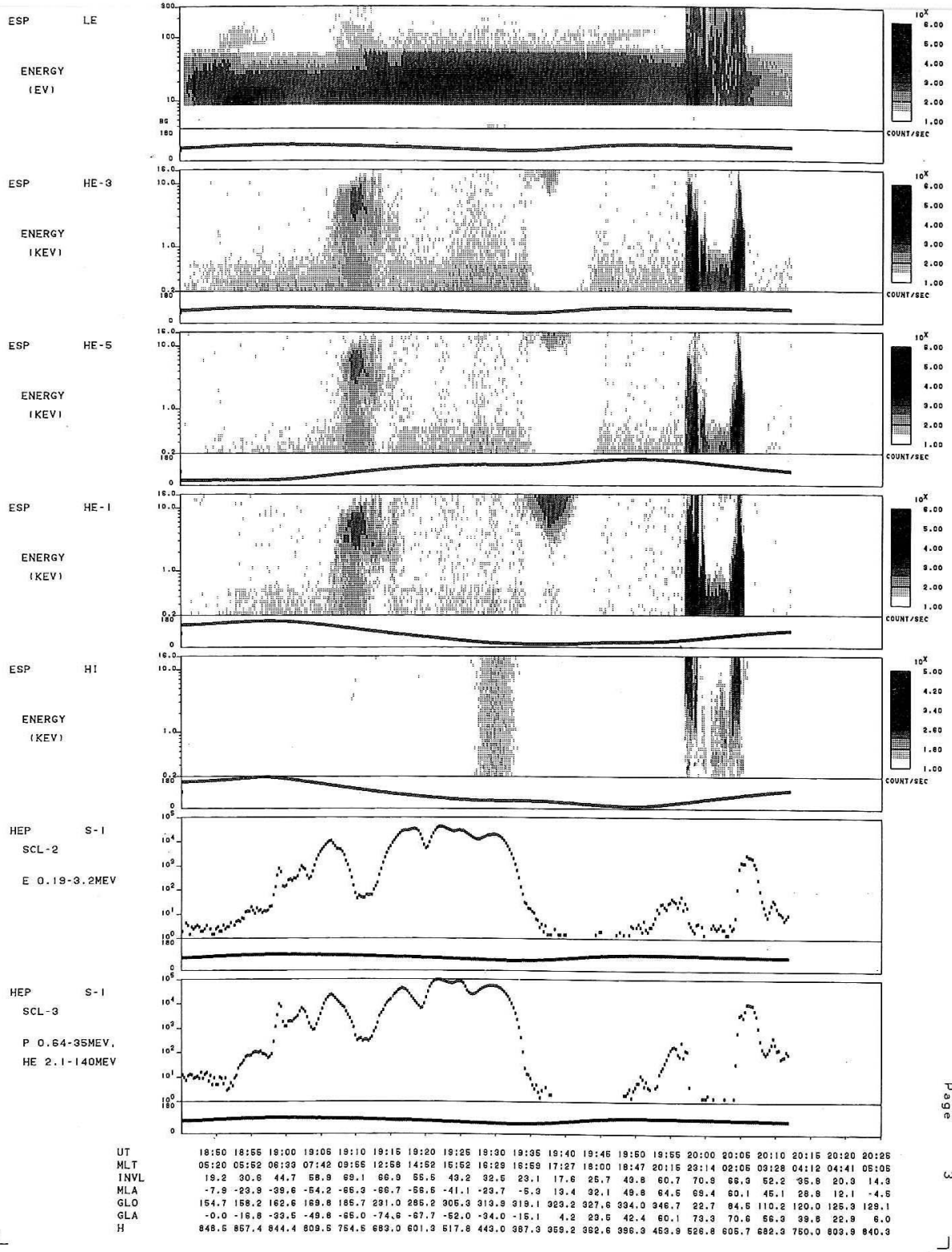

Fig. 8. Same as Fig. 7. Rev. No. is 00944. 


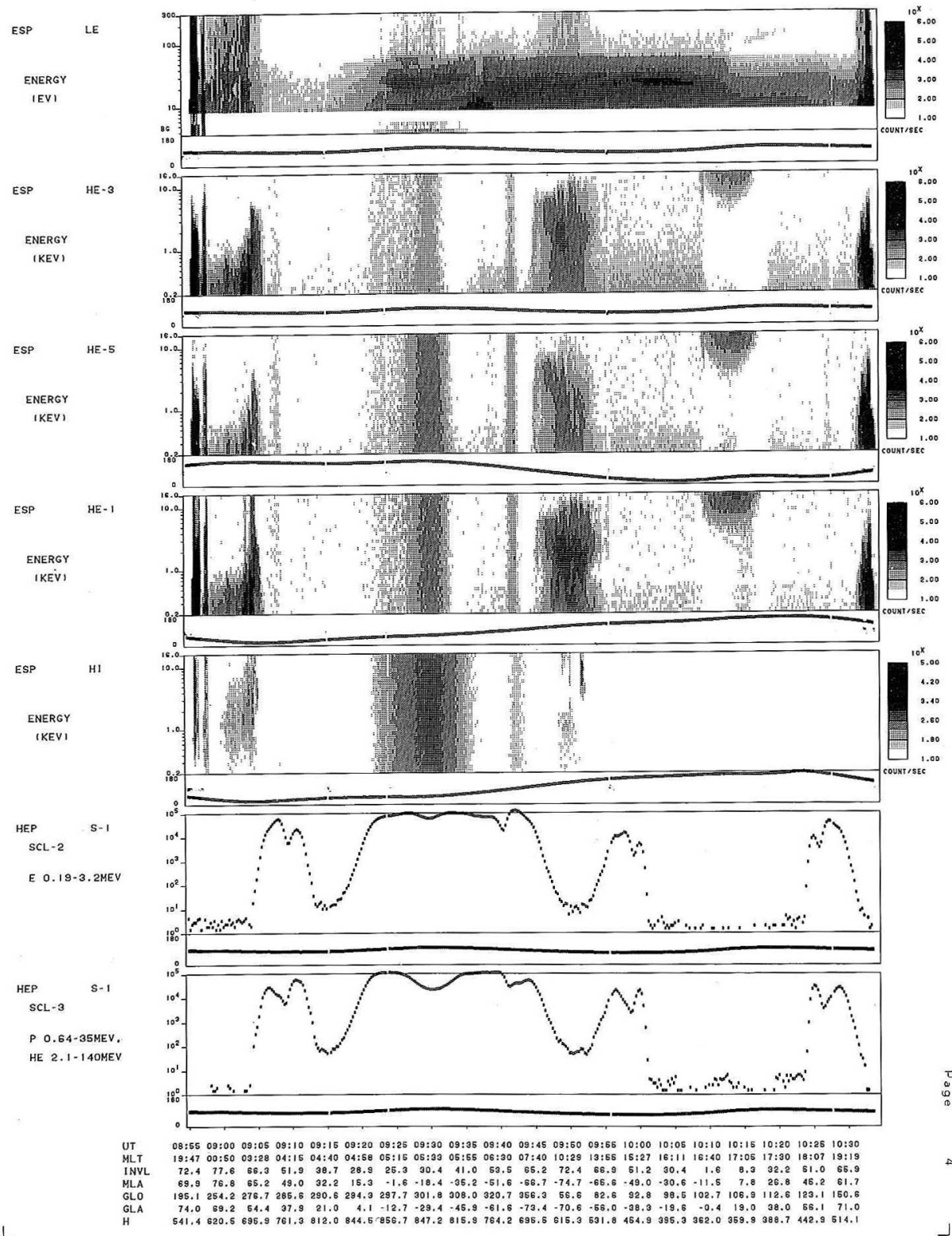

Fig. 9. Same as Fig. 7. Rev. No. is 00953. 
of the South-Atlantic geomagnetic anomaly, and the equator zone.

At first, let us discuss characteristics of auroral particles. It should be noted that in each figure the southern auroral region is in the dayside while the northern one is in the nightside, and that the highest invariant latitudes are $\left(72.5^{\circ}\right.$, $\left.73.7^{\circ}\right)$ at Rev. $00835,\left(70.0^{\circ}, 71.3^{\circ}\right)$ at Rev. 00944 and $\left(72.5^{\circ}, 78.2^{\circ}\right)$ at Rev. 00953, respectively, where the first and second values in the parenthesis are for the southern and northern hemispheres, respectively. Characteristics of auroral particles observed in these orbits are described below.

(a) The electron precipitation regions are observed at latitudes higher than the trapping boundary of higher-energy particles (see HEP data) in both dayside and nightside regions. In more detail, the nightside precipitation occurs just at the trapping boundary, whereas the dayside precipitation is found in a broader region.

(b) The electron precipitation pattern is diffuse in the dayside auroral region, whereas in the nightside it is quite discrete and the polar cap region is located between two discrete precipitation regions. The discrete precipitation in the nightside is composed of a few inverted- $V$ events.

(c) The pitch angle distribution of auroral electrons which can be obtained by comparison of HE-1 $\sim 5$ data is found to be widely spread in both discrete and diffuse precipitations, although the precipitating component is much more intense than the upward flux.

(d) The HI data show ion fluxes precipitating into the atmosphere. It can be seen that the ion spectrum is harder than the corresponding electrons.

The above characteristics are generally consistent with earlier satellite observations (e.g., Frank and Ackerson, 1971; Frank and Gurnett, 1971; MenG, 1978; MuKai and HiraO, 1982), but the feature (c) and (d) cannot be explained by a simple acceleration mechanism such as the electric double-layer.

Next, the observations of the South-Atlantic anomaly region are described. As can be seen in Fig. 9, in the midst of this region both HE and HI data have been contaminated by high-energy particles penetrating through the satellite and instrument structures, and the HEP instrument observed intense fluxes of high-energy particles simultaneously. Therefore, it seems difficult at present to obtain a meaningful value of the flux from the HE and HI data in this region. However, it can be seen in different satellite locations that similarly high levels of high-energy particle fluxes do not necessarily produce similar count rates in the HE and HI data. Spectral difference of high-energy particles may produce different background count rates in the ESP instrument. Future works through cooperative study with the HEP investigator group would make it possible to subtract reasonable background count rates from the HE and $\mathrm{HI}$ data in the South-Atlantic anomaly region. In other regions, there is no serious problem in subtraction of background count rates.

The HE data also show a peculiar feature that energetic electrons are observed at dusk hours near the equator in Figs. 8 and 9. These electrons are energetic, but since the HEP instrument have not detected these electrons, they may have 


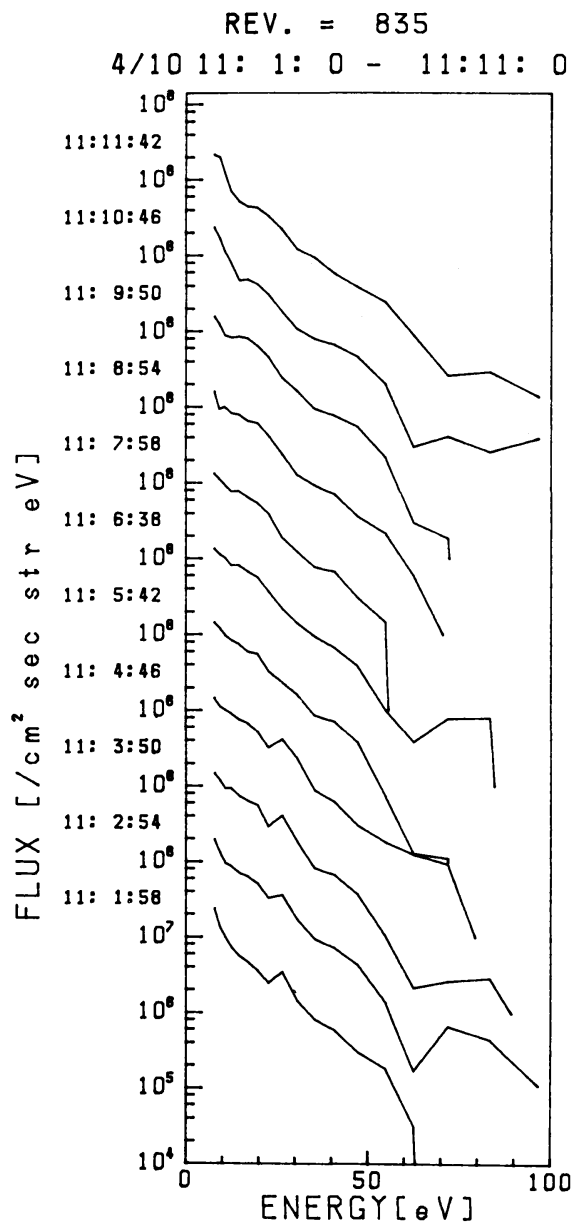

(a)

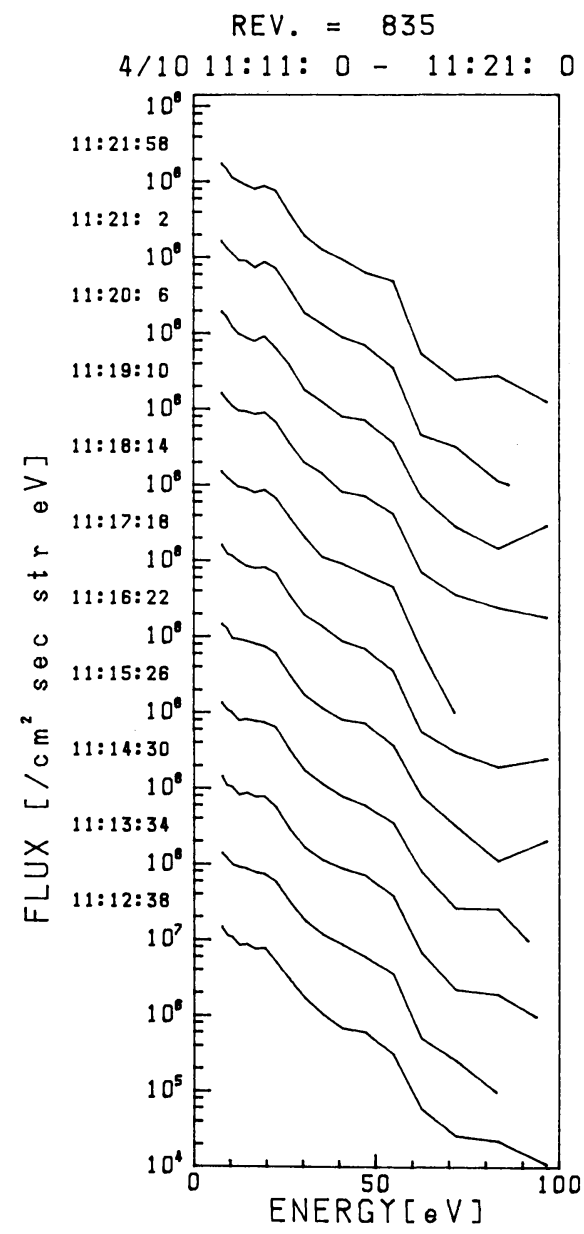

(b)

Fig. 10 .

energies of $10-100 \mathrm{keV}$. Voss and SMITH (1980) have surveyed global zones of energetic particle precipitation and found an equatorial zone of ions and neutrals with energies of $10-100 \mathrm{keV}$. Further work is required to delineate the above feature observed by the EXOS-C satellite.

Now we proceed to discuss characteristics of the LE data. The LE data show photoelectrons escaping from the topside ionosphere and also secondary electrons due to precipitation of auroral particles at high latitudes. The data below $6 \mathrm{eV}$ provide the background count rate as noted in Section 2 and are indicated in Figs. 7, 8 and 9, and the data in the energy range from 6 to 8.3 $\mathrm{eV}$ are not shown to avoid confusion (see Appendix). It can be seen that the photoelectron energy distribution is characterized by a sharp cutoff near $60 \mathrm{eV}$ 


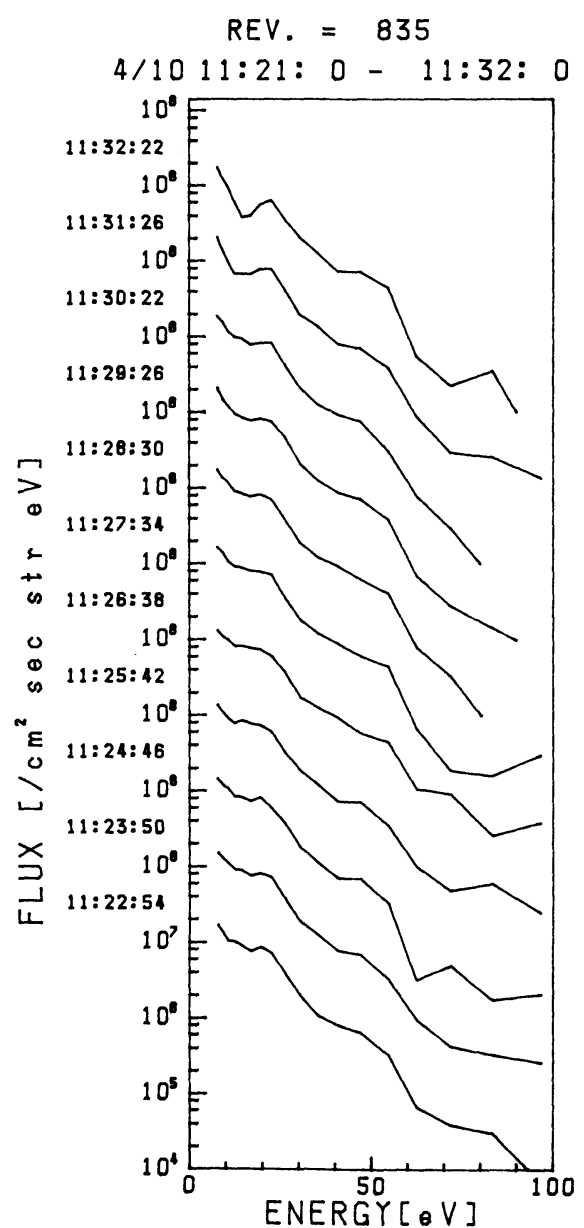

(c)

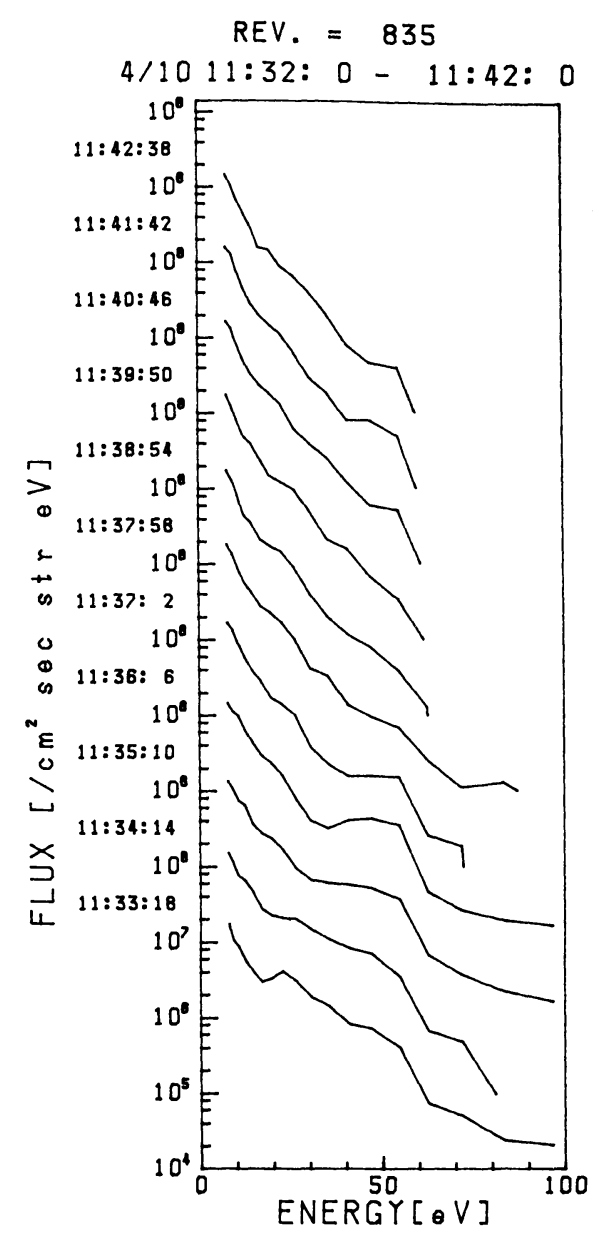

(d)

Fig. 10.

and also some structure in the energy range from 20 to $30 \mathrm{eV}$. This feature is shown more clearly in Fig. 10, in which the energy spectra are presented every 56 seconds in the time interval from 11:01 UT to 12:03 UT at Rev. 00835. The solar zenith angle $(\chi)$ at the satellite location has varied as $80^{\circ}(11: 01$ UT) $-79^{\circ}(11: 09$ UT $)-94^{\circ}(11: 40$ UT $)-101^{\circ}(11: 57$ UT $)-100^{\circ}(12: 03$ UT $)$. Note that the photoelectron flux decreased after $\sim 11: 40 \mathrm{UT}$ and also that the energy spectra include secondary electrons due to diffuse precipitation of auroral particles around 11:25 UT.

MUKAI and HiRAO $(1973,1975)$ originally found the existence of several peaks in the energy range from 20 to $30 \mathrm{eV}$ at lower altitudes, which could be attributed to photoelectrons emitted from $\mathrm{N}_{2}$ and $\mathrm{O}$ by the intense solar $\mathrm{He}$ II 


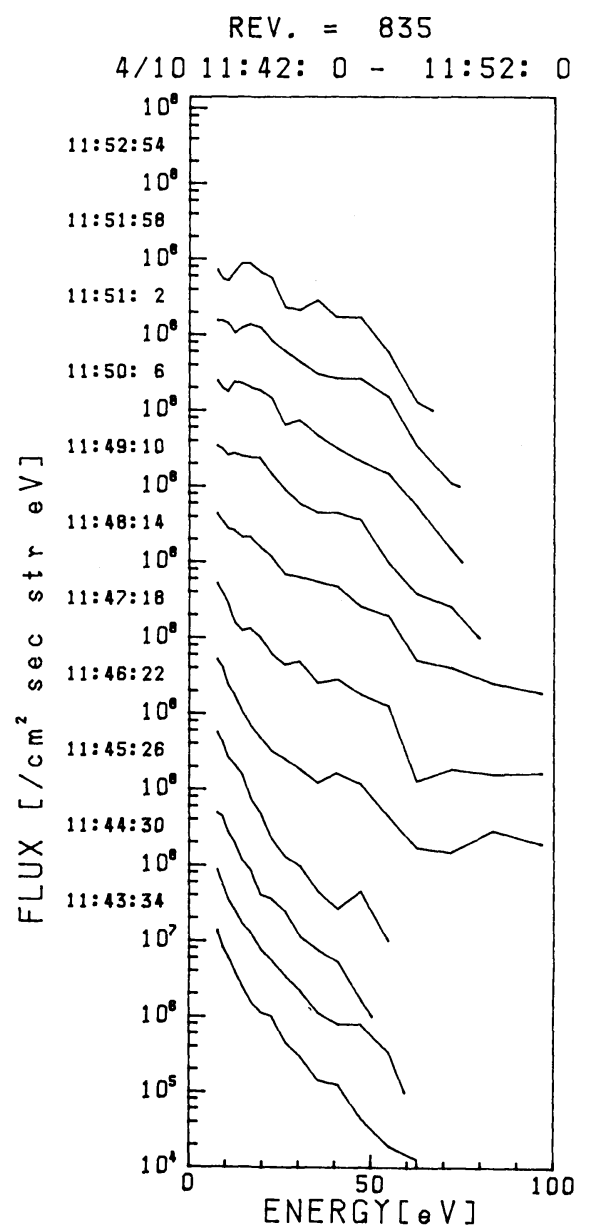

(e)

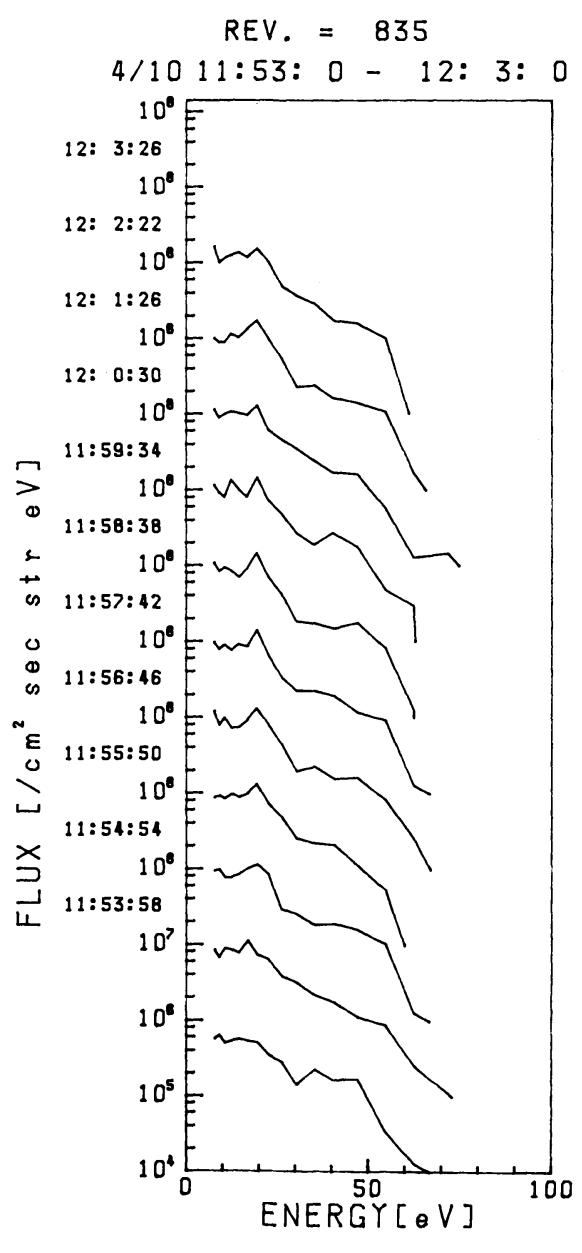

(f)

Fig. 10. Energy spectra of low-energy electrons observed at Rev. 00835 . The data at every 56 seconds are presented. Successive record is shifted up by an order of magnitude.

(304 A) radiation, and which were smeared out due to Coulomb collisions with ambient thermal electrons at higher altitudes. These peaks have been identified clearly also by satellite observations (DOERING et al., 1975, 1976; LEE et al., 1980a). The drop in the spectrum above $60 \mathrm{eV}$ which is due to the absence of significant solar radiation at energies higher than 70-80 eV has also been identified previously (MUKAI and HIRAO, 1975; DOERING et al., 1975, 1976; LEE et al., 1980a). Therefore, the features of the photoelectron energy distribution observed from the EXOS-C satellite are generally consistent with the previous results.

However, the structure in the energy range from 20 to $30 \mathrm{eV}$ looks highly variable. For example, a peak can be seen at the energy of $27 \mathrm{eV}$ in the first 
four spectra $\left(11: 01: 58 \sim 11: 04: 46 \mathrm{UT} ; \chi \approx 80^{\circ}\right)$. A plateau-like structure with a shoulder at $\sim 20 \mathrm{eV}$ can be seen in most spectra in Panel (b) and (c), and the shoulder sometimes changes into a broad peak. The spectra have no characteristic features during some periods of time after $\sim 11: 40$ UT $\left(\chi \approx 94^{\circ}\right)$, but a peak at $\sim 20 \mathrm{eV}$ can be seen again at the largest solar zenith angle $\left(\chi \approx 101^{\circ}\right)$ during 11:55 12:03 UT, though the electron flux has decreased about one order of magnitude below the daytime flux. LEE et al. (1980b) also have found that both the flux and spectral structure of photoelectrons in the energy range of 20 to $30 \mathrm{eV}$ are extremely variable at altitudes higher than $300 \mathrm{~km}$ and have a good correlation with the density of the ambient plasma. Unfortunately we have not obtained the electron density data in this particular orbit.

At Rev. 00953, the structure in the energy range of 20-30 eV has some peculiar feature. Figure 11 shows energy spectra during the period of 09:17 $\left(\chi=105^{\circ}\right)-09: 47\left(\chi=86^{\circ}\right)$ UT. The energy spectra after $\sim 09: 40$ UT $\left(\chi=93^{\circ}\right)$ are similar to those in the previous figures, but the peak observed during 09:23 $\left(\chi=104^{\circ}\right)-09: 34\left(\chi=98^{\circ}\right)$ UT over the South-Atlantic anomaly region is too prominent to be identified purely as ionospheric photoelectrons at these large solar zenith angles. The source of these electrons is not identified at present.

\section{Conclusion}

This paper has described characteristics of the ESP instruments on board the EXOS-C satellite ("OHZORA"), the technical performance in orbit, and a preliminary analysis of results which were obtained during the initial phase of satellite operation.

The ESP instrument consists of three sensors (LE, HE and HI) and a common electronics for control and processing of data. The LE sensor measures an energy spectrum of low-energy electrons over the energy range from $6 \mathrm{eV}$ to $300 \mathrm{eV}$, while the $\mathrm{HE}$ and $\mathrm{HI}$ sensors measure higher-energy electrons and positive ions, respectively, from $200 \mathrm{eV}$ to $16 \mathrm{keV}$. The LE sensor consists of an inlet collimator, a hemispherical electrostatic analyzer and a channelron. Several considerations were made in instrumental design for reliable measurement of lowenergy electrons. The HI sensor has a similar configuration to the LE sensor but with different geometrical dimension. The HE sensor consists of a sophisticated collimator, a quadrispherical electrostatic analyzer and five channeltrons in order to measure energy distributions of electrons at five pitch angles simultaneously. Characteristics of each sensor were calibrated in the pre-flight test by using the calibration facility at ISAS.

The technical performance of the instrument in orbit has been excellent. The ESP experiment has provided us with global characteristics of low-energy charged particle distribution over a wide range of latitude, longitude, altitude $(350-865 \mathrm{~km})$ and local time. The main scientific results are summarized as follows.

The HE and HI sensors have observed auroral particles. The precipitation 


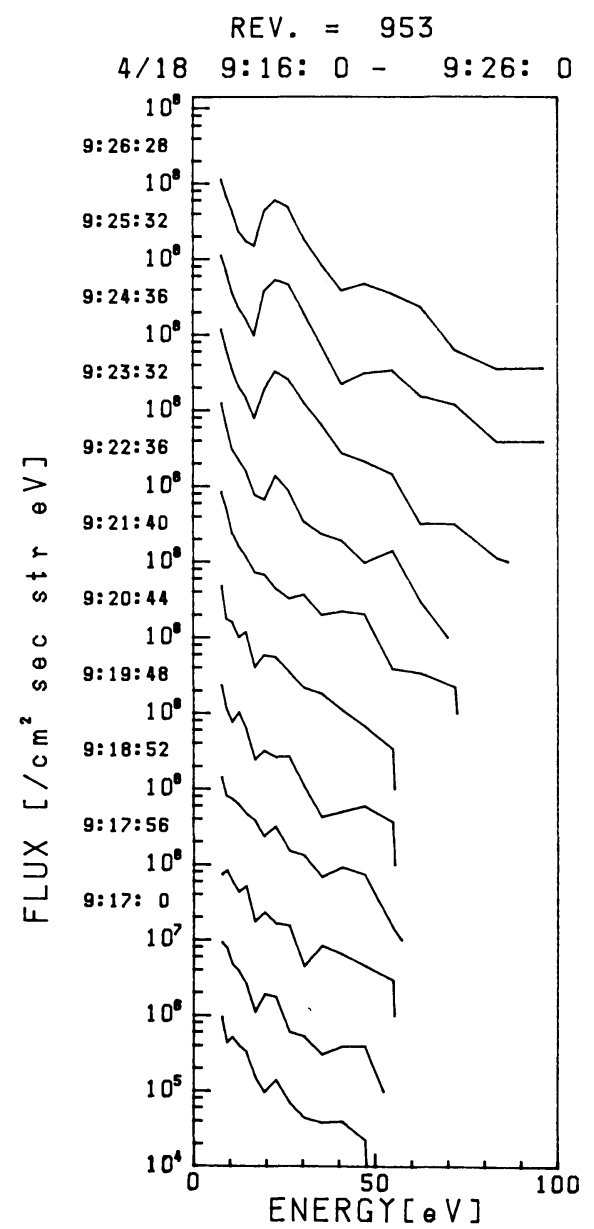

(a)

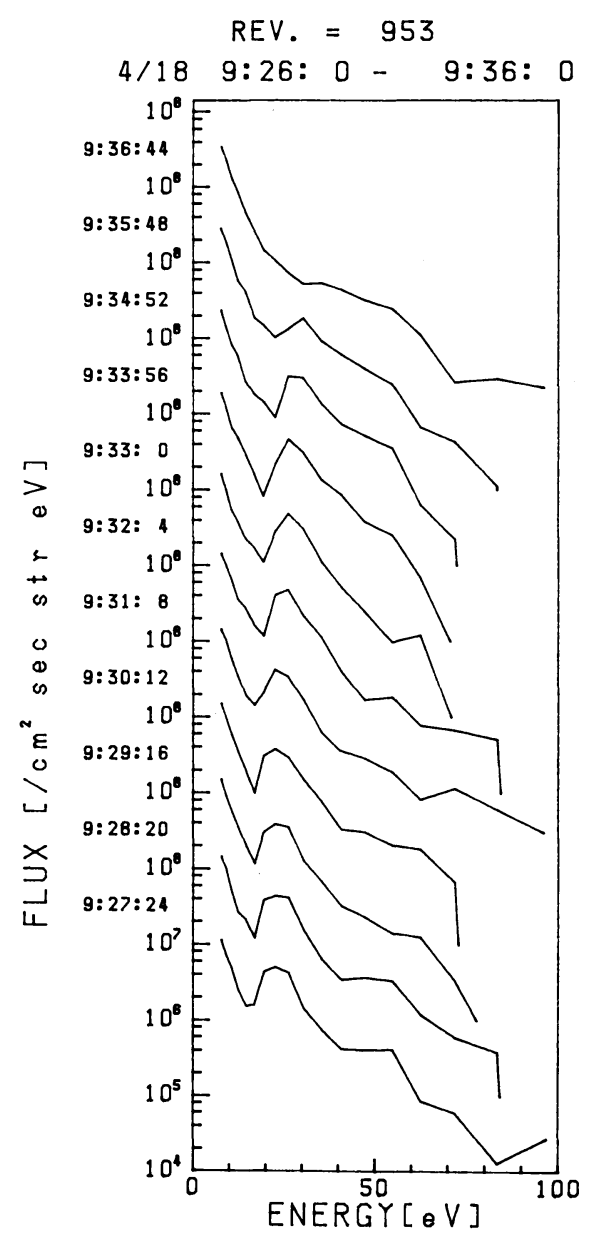

(b)

Fig. 11 .

of auroral particles are observed at latitudes higher than the trapping boundary of higher-energy particles. The electron precipitation pattern is diffuse in the dayside auroral region, whereas it is quite discrete on the nightside. The pitch angle distribution of precipitating auroral electrons is found to be rather widely spread in pitch angles in both discrete and diffuse precipitations. The energy spectrum of ions precipitating into the auroral region is found to be generally harder than the corresponding electrons.

Energetic electrons with energies of $10-100 \mathrm{keV}$ have been newly detected at dusk hours near the equator. The nature of these electrons is not yet known.

The photoelectron energy distribution is shown to have a peak in the energy range of 20 to $30 \mathrm{eV}$ and also a sharp cutoff near $60 \mathrm{eV}$. However, the spectral structure in the energy range of 20 to $30 \mathrm{eV}$ is found to make a significant 


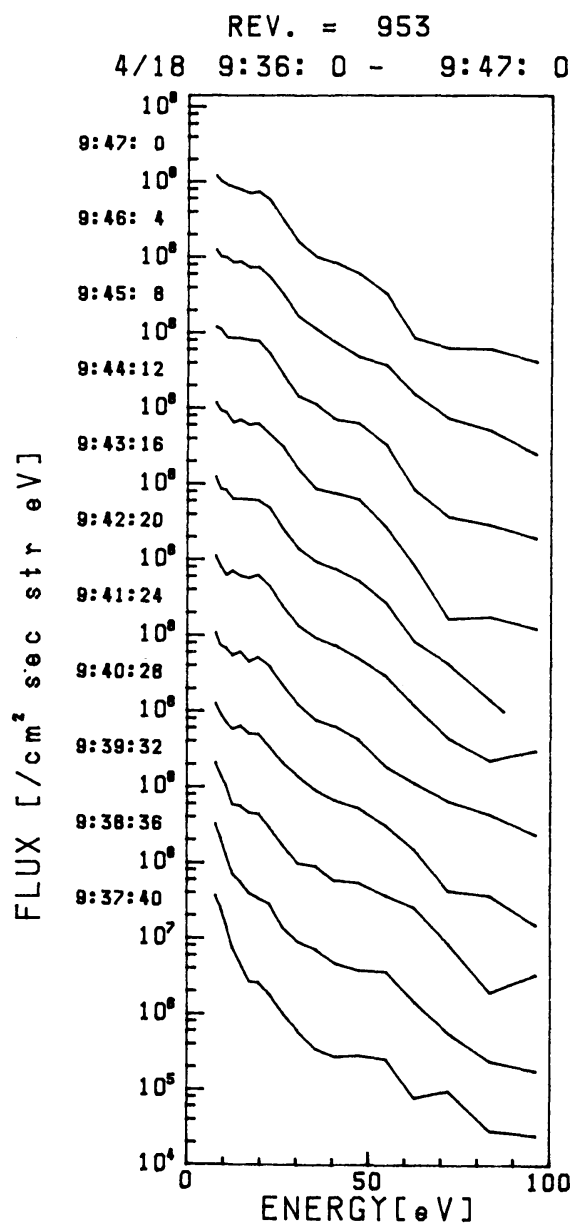

Fig. 11. Same as Fig. 10 at Rev. 00953.

(c)

variation depending on latitude, longitude and local time. A large peak at 20-30 $\mathrm{eV}$ which is too prominent to be identified as photoelectrons escaping from the ionosphere is observed occasionally over the South-Atlantic anomaly region.

Most of the above characteristics are generally consistent with earlier observations, and a further discussion will be reported in near future.

We would like to express our sincere thanks to the staff of the EXOS-C ("OHZORA") project for their extensive collaboration throughout whole phase of the project. We are also grateful to Dr. K. Nagata of Tamagawa University and "HEP" group for admission of using their data. We are also grateful to Dr. K. Oyama and Mr. K. Tanimoto for production of "Summary Plot". The sensor and electronic parts of the ESP instrument were manufactured by Mitaka-Koki Co., Ltd. and Shoei Electronic Co., Ltd., respectively. 


\section{APPENDIX}

The center energy, $E$ of particles which can pass through a hemispherical electrostatic analyzer is related with the analyzer parameters as

$$
E=q\left(V_{\mathrm{o}} R_{\mathrm{o}}^{2}-V_{\mathrm{i}} R_{\mathrm{i}}^{2}\right) /\left(R_{\mathrm{o}}^{2}-R_{\mathrm{i}}^{2}\right),
$$

where $q$ is particle charge and the analyzer parameters are defined in the text. If

$$
R_{\mathrm{i}} V_{\mathrm{i}}+R_{\mathrm{o}} V_{\mathrm{o}}=0
$$

then the electric potential at the center of the analyzer gap is equal to that of the inlet and exit slits, so that the fringing field effect is minimized.

From Eqs. (A1) and (A2)

$$
V_{\mathrm{o}}=(E / q)\left(R_{\mathrm{o}}-R_{\mathrm{i}}\right) / R_{\mathrm{o}}
$$

In our LE analyzer $\left(R_{\mathrm{o}}=28.5 \mathrm{~mm}, R_{\mathrm{i}}=21.5 \mathrm{~mm}\right)$

$$
V_{\mathrm{o}}=0.2456 \cdot(E / q)
$$

If $E$ and $V_{\mathrm{o}}$ are expressed in units of $\mathrm{eV}$ and volt, respectively,

$$
V_{\mathrm{o}}=-0.2456 \cdot E
$$

We have inserted a mesh between the exit slit and the channeltron. The particle energy must overcome the mesh potential, $V_{M}$ for being detected by the channeltron. That is,

$$
E+V_{\mathrm{M}}<0
$$

If

$$
V_{\mathrm{M}}=V_{\mathrm{o}}-5,
$$

then the electron energy should be greater than $6.6 \mathrm{eV}$, combining (A4)', (A5) and (A6). In practice this cutoff energy is not so clear because of the effect of the channeltron inlet potential $(\sim 200$ volts) penetrating through the rough mesh. Thus, the data obtained at energies which are marked by asterisks in Table 2 provide background information. In addition, the mesh potential is set at -80 volts at the step numbers 1 and 2 (see Table 2 ) in order to measure the background count rate more definitely. If $V_{M}=-80$ volts, the electron energy which satisfies the above conditions does not exist at all.

Electron trajectories, the energy of which is from $6 \mathrm{eV}$, to $8 \mathrm{eV}$, may be 
affected more or less by this mesh potential. Therefore, correction must be done for count rates obtained from $6 \mathrm{eV}$ to $8.3 \mathrm{eV}$, but it has not been made in this paper yet. The data in the above energy range are not presented in this paper to avoid confusion.

\section{REFERENCES}

Doering, J. P., W. K. Peterson, C. O. Bostrom, and J. C. A. Armstrong, Measurement of low-energy electrons in the day airglow and dayside auroral zone from Atmosphere ExplorerC, J. Geophys. Res., 80, 3934-3944, 1975.

Doering, J. P., W. K. Peterson, C. O. Bostrom, and T. A. Potemra, High resolution daytime photoelectron energy spectra from AE-E, Geophys. Res. Lett., 3, 129-131, 1976.

FRANK, L. A. and K. L. ACKERSON, Observations by Charged particle precipitation into the auroral zone, J. Geophys. Res., 76, 3612-3643, 1971.

FrANK, L. A. and D. A. GURNETT, Distribution of plasmas and electric fields over the auroral zones and polar caps, J. Geophys. Res., 76, 6829-6846, 1972.

KAYA, N., T. MUKaI, and H. MATSUMOTO, Characteristics of auroral particles observed by EXOSC, J. Geomag. Geoelectr., this issue, 347-363, 1985.

Lee, J. S., J. P. Doering, T. A. Potemra, and L. H. Brace, Measurements of the ambient photoelectron spectrum from Atmosphere Explorer: I. AE-E measurements below $300 \mathrm{~km}$ during solar minimum conditions, Planet. Space Sci., 28, 947-971, 1980a.

Lee, J. S., J. P. Doering, T. A. Potemra, and L. H. Brace, Measurements of the ambient photoelectron spectrum from Atmosphere Explorer: II. AE-E measurements from 300 to 1000 km during solar minimum conditions, Planet. Space Sci., 28, 973-996, 1980b.

MENG, C. I., Electron precipitation and polar aurora, Space Sci. Rev., 22, 223-300, 1978.

MUKAI, T. and K. HIRAO, Rocket measurement of the differential energy spectrum of the photoelectrons, J. Geophys. Res., 78, 8395-8398, 1973.

MUKAI, T. and K. HIRAO, Rocket measurement of the photoelectrons in the ionosphere by K-9M-47, Bull. Inst. Space Aeronaut. Sci., 11, 593-603, 1975.

MuKAI, T. and K. HIRAO, Characteristics of the inverted-V events observed by the KYOKKO satellite, Mem. Nat. Inst. Polar Res., Special Issue, 22, 116-124, 1982.

Voss, H. D. and L. G. SmITH, Global zones of energetic particle precipitation, J. Atmos. Terr. Phys., 42, 227-239, 1980. 